\title{
Búsqueda de primers y optimización de técnicas para la determinación de marcadores de ADN microsatélites en langostino argentino (Pleoticus muelleri)
}

\author{
Search for primers and optimization of determination techniques of ADN markers \\ microsatelites in argentinian shrimp (Pleoticus muelleri) \\ Estefanía Soledad GESTO, estefi_gesto@hotmail.com \\ Valeria MARCUCCI, valeriamarcucci@ hotmail.es \\ Pedro DE CARLI, pdecarli@uarg.unpa.edu.ar
}

Instituto de Ciencias del Ambientes, Sustentabilidad y Recursos Naturales. Universidad

Nacional de la Patagonia Austral, Unidad Académica Río Gallegos

Av. Gregores y Piloto “Lero" Rivera, Río Gallegos, Santa Cruz, Argentina

Recibido: 20/04/2018. Aceptado: 04/12/2018

\section{RESUMEN}

El langostino argentino (Pleoticus muelleri) se distribuye a lo largo de las costas del Océano Atlántico Sudoccidental, entre $\operatorname{los} 20^{\circ}$ y $50^{\circ}$ de Latitud S. En Argentina constituye en la actualidad uno de los recursos de mayor importancia para la actividad pesquera. El elevado valor comercial de este crustáceo en los mercados internacionales lo ha situado como uno de los principales productos de exportación pesquera argentina, representando una participación del $61 \%$ del total de exportaciones del sector, con un ingreso anual superior a los 1.200 millones de dólares estadounidenses.

En la actualidad, se dispone para la especie de un estudio genético restringido a la zona sur de su distribución (entre los 42 y $47^{\circ}$ de latitud S) y que analiza la diversidad de un segmento de ADN mitocondrial del gen COI, y otro que estudia la variabilidad genética del langostino $P$. muelleri en toda su área de distribución geográfica mediante el análisis de un fragmento de ADN mitocondrial correspondiente al gen región control (CR).

Este trabajo tiene como objetivo evaluar marcadores moleculares microsatélites como información de base para el estudio poblacional en el langostino argentino.

A partir de bibliografía se seleccionaron cuatro (4) primers que permitieron amplificar microsatélites en otras especies de la misma familia (Solenoceridae).

Solo se han obtenidos resultados de amplificación a partir de un par de iniciadores (ZG-71). La secuenciación de estos fragmentos no permitió detectar la conservación de los microsatélites esperados para el desarrollo del marcador específico.

Palabras clave: crustáceo, genética poblacional, microsatélite, recurso pesquero.

\begin{abstract}
The Argentine shrimp (Pleoticus muelleri) is distributed along the coasts of the South West Atlantic Ocean, between $20^{\circ}$ and $50^{\circ}$ of Latitude S. In Argentina, it is currently one of the most important resources for fishing activity. The high commercial value of this crustacean in
\end{abstract}


the international markets has placed it as one of the main export products of Argentina, representing a participation of $61 \%$ of the total exports of the sector, with an annual income of more than 1,200 million US Dollars.

Currently, a restricted genetic study is available for the species to the south of its distribution (between 42 and $47^{\circ}$ latitude $S$ ) and it analyzes the diversity of a mitochondrial DNA segment of the COI gene, and another that studies the genetic variability of $P$. muelleri shrimp throughout its geographic range by analyzing a fragment of mitochondrial DNA corresponding to the control region (CR) gene.

The objective of this work is to evaluate microsatellite molecular markers as basic information for the population study of Argentine shrimp.

From the bibliography, four (4) primers were selected that allowed to amplify microsatellites in other species of the same family (Solenoceridae).

Only amplification results have been obtained from a pair of primers (ZG-71). The sequencing of these fragments did not allow to detect the conservation of the microsatellites expected for the development of the specific marker.

Keywords: crustacean, population genetics, microsatellite, fishery resource.

\section{INTRODUCCIÓN}

El langostino argentino Pleoticus muelleri (Bate, 1888) se distribuye a lo largo de las costas del Océano Atlántico desde Espíritu Santo, Brasil $\left(20^{\circ} \mathrm{S}\right)$, hasta las costas de Santa Cruz, Argentina (50 S) (Boschi et al., 1992). Es sin dudas uno de los recursos de mayor importancia para la historia del desarrollo de la actividad pesquera en Argentina. En la década de 1980 la actividad inicia en Patagonia y a partir de la década de 1990 el $96 \%$ del desembarque declarado de esta especie proviene de dicha pesquería. Su elevado valor comercial en los mercados internacionales lo ha situado como uno de los principales productos de exportación pesquera argentina, representando para el año 2017 el $61 \%$ del total de exportaciones del sector, y un ingreso anual superior a los 1.200 millones de dólares estadounidenses (DEP-SPyA, 2018).

Junto con el desarrollo de la pesquería se da inicio a la investigación del recurso y en el año 1984 la provincia de Santa Cruz establece la primera zona de veda total en Mazarredo al sur del Golfo San Jorge, para proteger los fondos de cría y crecimiento de langostino. En el año 1988 las provincias de Chubut y Santa Cruz firman un Convenio de Administración Conjunta declarando al Golfo San Jorge como una unidad biológica y económica para la explotación del recurso langostino, y en la década de 1990 la Subsecretaría de Pesca de Santa Cruz estableció un sistema de manejo adaptativo, mediante el empleo de áreas de veda dinámicas con el objetivo de evitar la sobrepesca del crecimiento. Las capturas anuales de langostino presentan importantes variaciones en sus números, con mínimos en los años 1987, 1995 y 2005 (Fischbach et al., 2006). A partir del año 2008, con una captura total anual de $47.400 \mathrm{t}$, la actividad comienza a concentrar su capacidad pesquera en aguas de jurisdicción nacional y se inicia un proceso de reducción del esfuerzo pesquero en aguas del Golfo San Jorge. De ahí en más, las capturas totales se incrementan anualmente de manera sostenida, hasta alcanzar en el año 2017 un total de 241.500t (MINAGRI, 2018).

La caracterización de la estructura genética poblacional es particularmente importante para el manejo y conservación de especies de importancia ecológica y con potencial pesquero (McMillen-Jackson et al., 1994). En el ambiente marino, el flujo génico o movimiento de 
genes entre poblaciones ocurre a través de la dispersión de larvas y adultos (Slatkin, 1985). En especies pelágicas y demersales se ha encontrado una débil estructuración genética poblacional, incluso a través de grandes distancias geográficas (Ward et al., 1994). En el caso del langostino, los huevos se liberan al mar y tienden a ser bentónicos, pero las larvas son planctónicas, y su posición en la columna de agua varía según la luminosidad, turbulencia y transparencia (Boschi, 1989). Cuando las barreras para la migración son inexistentes, especies con alta capacidad de dispersión y tamaños poblacionales grandes, podrían representar idealmente una única población genéticamente homogénea (panmíctica) con un alto flujo genético (Machado Schiaffino et al., 2011; Palumbi, 1994). Sin embargo, Machado Schiaffino y colaboradores (2011) menciona que organismos con gran capacidad de dispersión, pueden exhibir una estructura genética poblacional determinada por el ambiente o por la historia de vida de la especie. El análisis de la estructura genética poblacional constituye una herramienta importante en el manejo de invertebrados marinos bajo explotación (Thorpe et al., 2000), y su identificación puede requerir modificar las estrategias de manejo.

Varias especies de crustáceos peneidos sostienen pesquerías de importancia a nivel mundial, por lo que la mayoría de los estudios genéticos publicados sobre este grupo buscan determinar estructuras de stock pesquero (Benzie, 2000). La poca variabilidad registrada mediante estudios que analizaron la estructura geográfica de las poblaciones mediante alozimas (Hedgecock et al., 1982), sumado al creciente interés en la acuicultura de camarones peneidos ha llevado al desarrollo de estudios de marcadores de ADN con mayor variabilidad, tales como mitocondrial (ADNmt) y microsatélites (Stepien y Kocher, 1997). Los resultados obtenidos en poblaciones de Penaeus monodon de Australia del Oeste a partir de alozimas (Benzie et al., 1992), ADNmt (Benzie et al., 2002) y microsatélites (Broker et al.,1999) han permitido demostrar que son significativamente diferentes de otras poblaciones de Australia. Los datos de ADNmt sugieren además que la población de Australia occidental es diferente de las poblaciones de Indonesia (Benzie et al., 2002).

En los estudios de diversidad genética, los marcadores más utilizados son los microsatélites o secuencias simples repetidas (SSRs; FAO, 2010). Se caracterizan por ser altamente polimórficos en cuanto a su longitud: por lo tanto, son regiones adecuadas para usarse como marcadores moleculares a nivel poblacional (Zane et al., 2002). Sin embargo, su utilización es a veces compleja debido a la dificultad de desarrollarlos de novo para la especie en estudio, ya que el procedimiento convencional resulta muy laborioso y costoso (Malausa et al., 2011).

Existen pocos estudios genéticos realizados sobre $P$. muelleri. Las primeras secuenciaciones de ADN se efectuaron con el objetivo de identificar especies de crustáceos en alimentos; se utilizaron secuencias parciales de ADNmt como herramienta de autenticidad alimentaria (Calo-Mata et al., 2009). De Carli (2012), realizó un estudio de distribución de la variabilidad genética para esta especie pero fue insuficiente para poner a prueba la existencia de estructuración genética, ya que sólo se investigó para una región del gen citocromo oxidasa subunidad I (COI) de individuos pertenecientes a localidades en el extremo sur de su rango de distribución. Posterior a ello, Marcucci y colaboradores (2017), analizó la variabilidad genética del langostino $P$. muelleri en toda su área de distribución geográfica mediante el análisis de un fragmento de ADN mitocondrial correspondiente a la región control (CR). Los índices analizados indicaron que $P$. muelleri es una gran población panmíctica con un alto grado de flujo génico. Sin embargo no haber detectado una clara estructuración genética con el marcador analizado no implicaría necesariamente que fuera inexistente, por lo que propone corroborar los resultados obtenidos y poner a prueba la existencia de diferencias entre los sitios geográficos propuestos analizando marcadores moleculares como lo microsatélites, dado que con ellos ha sido posible evidenciar la existencia de una estructura genética en las 
poblaciones de otras especies de peneidos, cuando su análisis mediante otros marcadores no permitía detectarlo (Liu et al., 2004).

Este trabajo tiene como objetivo evaluar marcadores moleculares microsatélites que permitan aportar conocimiento de base para el estudio poblacional en el langostino argentino, que sea de utilidad para diseñar estrategias de manejo sustentable y conservación.

\section{MATERIALES Y MÉTODOS}

Este estudio se realizó a partir de material disponible en la colección de tejido del laboratorio de Genética Pesquera de la Universidad Nacional de la Patagonia Austral. La colección se compone de tejido muscular conservado en etanol (96\%) proveniente de capturas comerciales obtenidas del golfo San Jorge, litoral de Rawson, golfo San Matías, litoral de Uruguay y Brasil (Rio Grande do Sul, Santa Catarina y Río de Janeiro).

Se extrajo el ADN total (ADNt) de individuos provenientes de cada localidad. Para ello se utilizó el método de extracción salina propuesto por Aljanabi y Martinez (1997) con modificaciones.

Se seleccionaron cuatro (4) iniciadores (ZG-29, ZG-66, ZG-71 y ZG-85; ver Tabla 1) que permitieron amplificar microsatélites en otras especies de la misma familia (Solenoceridae) y cuyos accesos en GenBank son JQ954752, JQ954738, JQ954740 y JQ954742 (Liu et al. 2012). La reacción en cadena de la polimerasa se llevó a cabo en un termociclador MultiGene ${ }^{\mathrm{TM}}$ OptiMax (LabNet). La reacción de amplificación se preparó a volumen final de $20 \mu$, conteniendo Taq polimerasa (1U, PBL), $\mathrm{MgCl}_{2}(1,5 \mathrm{mM})$, buffer para PCR (1X), dNTP $(0,2 \mathrm{mM}), 2$ primers $(0,2 \mu \mathrm{M})$, extracto de ADNt $(1 \mathrm{ng})$ y agua ultrapura. El protocolo de ciclado se presenta en la Tabla 1 para cada primer.

Tabla 1 - Primers y condiciones de PCR (Liu et al., 2012).

\begin{tabular}{|c|c|c|c|c|}
\hline Primer & Secuencia & $\begin{array}{l}\text { Motivo de } \\
\text { repetición }\end{array}$ & $\begin{array}{c}\mathbf{T m} \\
\left({ }^{\circ} \mathbf{C}\right)\end{array}$ & Protocolo de Ciclado \\
\hline ZG29 & $\begin{array}{c}\text { F: GATATGGCGGTTGAGTGA } \\
\text { R: } \\
\text { TACGTGGTTTATGTTGCTTA }\end{array}$ & $(\mathrm{AC})_{17}$ & \multirow{3}{*}{55} & 5 min a $94^{\circ} \mathrm{C}$ \\
\hline ZG66 & $\begin{array}{c}\text { F: TTCCATCCTATTTCTACTG } \\
\text { R: } \\
\text { ATAAGACGTTTACCTACAT }\end{array}$ & $(\mathrm{AC})_{14}$ & & $\begin{array}{l}45 \text { seg. a } 94^{\circ} \mathrm{C} \\
45 \text { seg a } \mathrm{Tm}\end{array}$ \\
\hline ZG85 & $\begin{array}{c}\mathrm{F}: \\
\text { AATGACAACTCTACAGGCTA } \\
\text { R: TCAATTCCAAGTGAATGC }\end{array}$ & $(\mathrm{TG})_{51 \ldots(\mathrm{GT})_{6}}$ & & $\begin{array}{l}\text { ciclos } \\
45 \mathrm{seg} . \text { a } 72^{\circ} \mathrm{C}\end{array}$ \\
\hline ZG71 & $\begin{array}{c}\text { F: } \\
\text { AAAGGCTGAAATCAAGAAG } \\
\text { R: } \\
\text { GAGGAAGAATGAGCGTTAG }\end{array}$ & $(\mathrm{CA})_{61}$ & 45 & $10 \min$ a $72^{\circ} \mathrm{C}$ \\
\hline
\end{tabular}

Se realizó la optimización del protocolo de PCR mediante ensayo de gradiente térmico y concentración de cloruro de magnesio.

Los productos de la amplificación se purificaron mediante kit de columnas (BIONER) en un volumen final de recuperación de $30 \mu \mathrm{l}$. La secuenciación de los productos de amplificación 
purificados se realizó mediante secuenciación automática empleando los primers ZG71-F y ZG71-R (Macrogen Inc. Korea).

Las secuencias obtenidas fueron alineadas en BioEdit versión 7.0. (Hall, 1999), Clustal W versión 2.0 (Thompson et al., 1994). Luego de ello estas secuencias fueron analizadas mediante Blastn para su verificación (http://www.ncbi.nlm.nih.gov/blast/Blast.cgi).

\section{RESULTADOS}

La extracción de ADN mediante el método de extracción salina propuesto por Aljanabi y Martinez (1997) dio resultados positivos.

Como resultado de la amplificación de ADN de langostino Pleoticus muelleri con los cebadores de la Tabla1 (Liu et al., 2012) se obtuvieron productos amplificados solo con el par de primers correspondientes a ZG-71. Al realizar el gradiente de temperatura se determinó $42,9^{\circ} \mathrm{C}$, como la temperatura óptima (Figura 1). La concentración de $\mathrm{MgCl}_{2}$ en la que el sistema funcionó mejor fue a de $1,5 \mathrm{M}$ a una temperatura de $43^{\circ} \mathrm{C}$ (Figura 2).

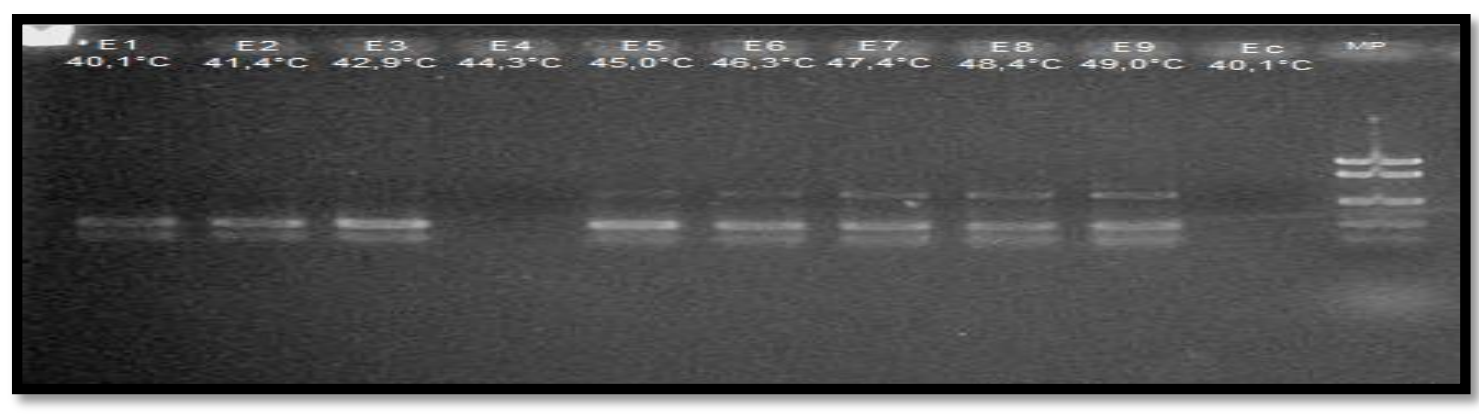

Figura 1 - Electroforesis Agarosa 3\%, 75V, 90min. PCR de gradiente (ZG71 F/R). Extracción salina. (MP: marcador de peso Ladder de masa precision, de 2000 pb a 100 pb.).

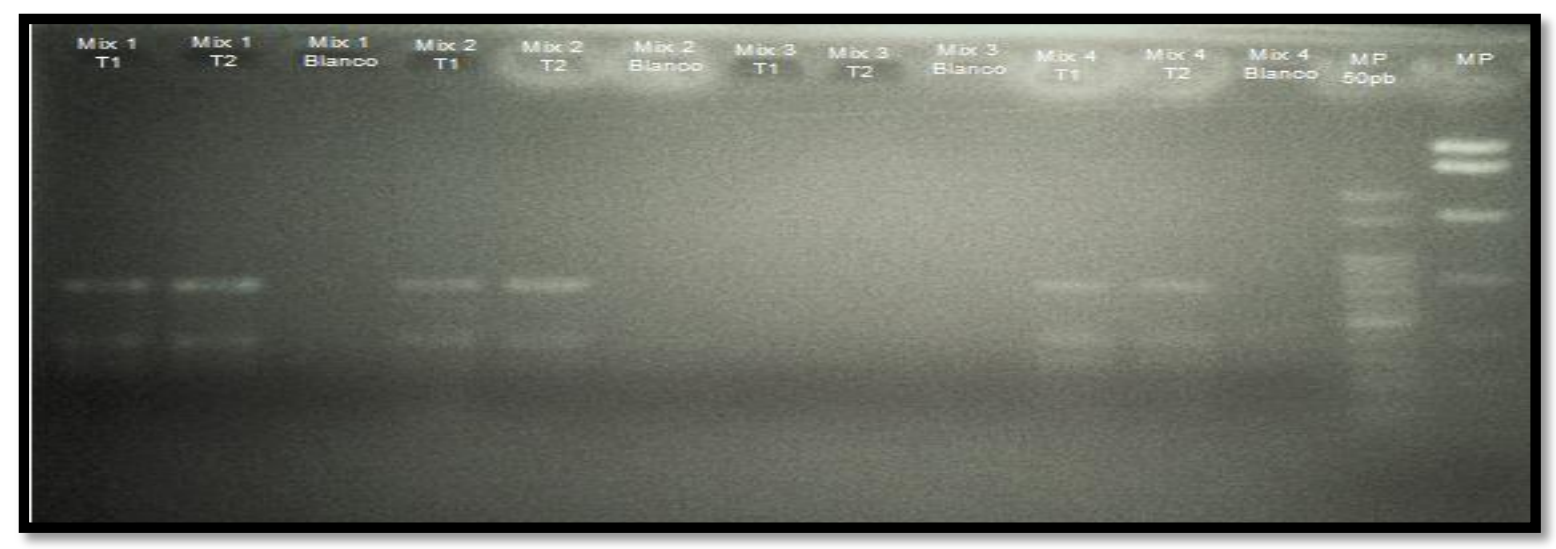

Figura 2 - Electroforesis Agarosa 3\%, 75V, 90min. PCR de gradiente con ZG71 F/R. (T1: $41^{\circ} \mathrm{C}$; T2: $43^{\circ} \mathrm{C}$; $\mathbf{M P}$ : marcador de peso Ladder de masa precision; MP 50pb: marcador de peso. Mix 1: reacción de amplificación con concentraciones de 1,5 $\mathrm{M}$ de $\mathrm{MgCl}_{2}$. Mix 2: reacción de amplificación con concentraciones de $2 \mathrm{M} \mathrm{de} \mathrm{MgCl}_{2}$.

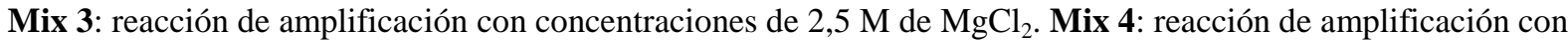
concentraciones de $3 \mathrm{M}$ de $\mathrm{MgCl}_{2}$ ).

El resultado de la amplificación obtenido podría corresponder a un individuo heterocigota para el marcador, con registros de aproximadamente 400 pb y 200 pb, o bien a una hibridación inespecífica de los primers (Figura 1 y 2). 
De la edición de las secuencias forward y reverse se obtuvo un fragmento de 334 pb de buena calidad sin incluir ningún gap, que pudo alinearse con el par de primers desarrollados para $S$. crassicornis y correspondientes al segmento ZG-71. Sin embargo el segmento obtenido no contiene la serie de repeticiones (CA) que se esperaba encontrar. El análisis mediante Blastn de la secuencia obtenida no permitió corroborar su identidad.

\section{CONCLUSIONES Y PERSPECTIVAS FUTURAS}

La utilización de marcadores microsatélites es a veces compleja y el procedimiento convencional resulta muy laborioso y costoso (Malausa et al., 2011). En este trabajo se pretendió en principio identificar marcadores microsatélites para $P$. muelleri de una manera económica, a partir de otros desarrollados en especies filogenéticamente emparentadas (Solenocera crassicornis). A pesar de haberse encontrado un par de primers que permitieron obtener un producto de amplificación, no fue posible detectar el microsatélite con el motivo de repetición deseado (CA) en la secuencia, por lo que se puede concluir que este primer no es de utilidad para los fines propuestos.

Ante estos resultados, se considera utilizar a futuro nuevas técnicas de secuenciación masiva (NGS) que permitan desarrollar nuevos primers para el aislamiento de microsatélites (Ekblom y Galindo, 2011; Malausa et al., 2011). La amplificación de los microsatélites se llevaría a cabo a partir de los cebadores diseñados desde secuencias de 5-10 individuos provenientes de distintas poblaciones de langostino alejadas geográficamente.

Estas técnicas de secuenciación masiva ya han sido utilizadas con éxito en otras especies de crustáceos. Perina y colaboradores (2016) desarrolló y caracterizó marcadores microsatélite en el camarón Palaemon serratus, y luego González Castellano (2016) utiliza éstos para ser tomados como punto de partida para el desarrollo de microsatélites de Palaemon elegans (Rathke, 1837). Por otro lado, Díaz Cabrera y colaboradores (2017), utilizaron la técnica NGS como herramienta para estudiar al crustáceo Panulirus pascuensis y obtuvo un total de 16 marcadores microsatélites a partir de 18 individuos, 9 de los cuales eran polimórficos, con el objetivo de aportar información de base que permitiera a futuro estudiar patrones de migración, estructura de la población y la diversidad genética de la especie en cuestión, permitiendo diseñar e implementar un adecuado manejo de la industria pesquera. Recientemente, con el avance de las tecnologías NGS y considerando que los marcadores microsatélites son herramientas efectivas para estudiar conectividad demográfica y detectar estructura geográfica de diversas especies marinas, Paz García y colaboradores (2017) ha reportado los primeros 32 microsatélites aislados de novo, que se amplificaron y genotipificaron con éxito para Lutjanus peru, también a partir de análisis de resultados de NGS.

Se espera que el análisis con marcadores microsatélites permita avanzar en el estudio del langostino $P$. muelleri a fin de evaluar si es posible identificar subpoblaciones genéticas, del mismo modo que ha sido determinado para otras especies marinas (Liu et al., 2004).

\section{BIBLIOGRAFÍA}

ALJANABI M. S. Y I. MARTÍNEZ. (1997). Universal and rapid salt-extraction of high quality genomic DNA for PCR-techniques. Nucleic acids research. 25(22): 4692-4693. https://doi.org/10.1093/nar/25.22.4692 
BATE C. S. (1888). Report on the Crustacea Macrura collected by H.M.S. Challenger during the years 1873-1876. Report on the Scientific results of the Voyage of H.M.S. Challenger during the years 1873-76. Zoology. 24: 942.

BENZIE J. A. H. (2000). Population genetic structure in penaeid prawns. Aquaculture Research. 31: 95-119. https://doi.org/10.1046/j.1365-2109.2000.00412.x

BENZIE J. A. H., BALLMENT E., FORBES A. T., DEMETRIADES N. T., SUGAMA K., HARYANTI Y S. MORIA. (2002). Mitochondrial DNA variation in Indo-Pacific populations of the giant tiger prawn, Penaeus monodon. Molecular Ecology. 11: 25532569. https://doi.org/10.1046/j.1365-294X.2002.01638.X

BENZIE J. A. H., FRUSHER S. Y E. BALLMENT. (1992). Geographical variation in allozyme frequencies of Penaeus monodon (Crustacea: Decapoda) populations in Australia. Australian Journal of Marine and Freshwater Research. 43: 715-725. https://doi.org/10.1071/MF9920715

BOSCHI E. E. (1989).Biología pesquera del langostino en el litoral patagónico de Argentina. Contribución INIDEP Nº 646 - Mar del Plata. Argentina. 71.

BOSCHI E., FISCHBACH C. Y M. IORIO. (1992). Catálogo ilustrado de los crustáceos estomatópodos y decápodos marinos de Argentina. Frente Marít. 10(A): 7- 94.

BROKER A.L., BENZIE J. A. H., BLAIR D. Y J. J. VERSINI. (1999). Population structure of the giant tiger prawn Penaeus monodon in Australian waters, determined using microsatellite markers. Marine Biology. 136: 149-157. https://doi.org/10.1007/s002270050017

CALO-MATA P., PASCOAL A., FERNÁNDEZ-No I., BÖHME K., GALLARDO J. M. Y J. BARROS VELÁZQUEZ. (2009). Evaluation of a novel 16S rRNA/tRNAVal mitochondrial marker for the identification and phylogenetic analysis of shrimp species belonging to the superfamily Penaeoidea. Analytical Biochemistry. 391(2): 127-134. https://doi.org/10.1016/j.ab.2009.05.020

DE CARLI P. (2012). Pesquería del langostino (Pleoticus muelleri, Bate 1888) en patagonia argentina: estructura genética poblacional y manejo sustentable. Tesis de Maestria. Universidad de Magallanes. Punta Arenas, Chile.

DEP-SPYA. (2018). Informe de Coyuntura. Enero 2018. Dirección de Economía Pesquera. Subsecretaría de Pesca y Acuicultura. Ministerio de Agricultura, Ganadería y Pesca. Argentina. 20.

DÍAZ CABRERA E., MEERHOFF E., ROJAS HERNANDEZ N., VEGA RETTER C. Y D. VELIZ. (2017). Development and characterization of the first 16 microsatellites loci for Panulirus pascuensis (Decapoda: Palinuridae) from Easter Island using Next Generation Sequencing. Biología Marina y Oceanografía. 52(2): 395-398. https://doi.org/10.4067/S0718-19572017000200018

EKBLOM R. Y J. GALINDO. (2011). Applications of next generation sequencing in molecular ecology of non- model organisms. Heredity. 107: 1-15. https://doi.org/10.1038/hdy.2010.152

FAO, DEPARTAMENTO DE AGRICULTURA. (2010). La situación de los recursos zoogenéticos mundiales para la alimentación y la agricultura. Comisión de Recursos Genéticos para la Alimentación y la Agricultura. Organización de las Naciones Unidas para la Agricultura y la Alimentación.Roma. 24.

FISCHBACH C., DE LA GARZA J. Y D. BERTUCHE. (2006). La pesquería del langostino patagónico en el período 1991-2005. Informe Técnico INIDEP. 03: 21. 
GONZÁLEZ CASTELLANO I. (2016). Análisis de marcadores moleculares en el camarón Palaemon elegans Rathke, 1837. Tesis de Maestría. Universidad Da Coru-a. Facultad de Ciencias.

HALL T. (1999). BioEdit: a user-friendly biological sequence alignment editor and analysis program for Windows 95/98/NT. Nucleic Acid SympSer. 41: 95-98.

HEDGECOCK D., TRACEY M. L. Y K. NELSON. (1982). Genetics. In: The biology of crustacean (ed. L. G. Abele). Academic Press, New York. 284-403.

IORIO M. I., SCELZO M. A. Y E. E. BOSCHI. (1990). Desarrollo larval y postlarval de langostino Pleoticus muelleri Bate, 1888 (Crustacea, Decapoda, Solenoceridae). Scientia Marina. 54(4): 329-341.

KANG J. H., PARK J. Y. Y H. S. JO. (2012). Rapid Development of Microsatellite Markers with 454 Pyrosequencing in a Vulnerable Fish, the Mottled Skate, Raja pulchra. International Journal of Molecular Sciences. 13: 7199-7211. https://doi.org/10.3390/ijms13067199

LIU P., MENG X.H., KONG J., ZHUANG Z.M., MA C.Y. Y Q. Y. WANG. (2004). Genetic diversity analysis of Penaeus chinensis by microsatellite DNA. Pro. Nat. Sci. 14(3), 333-338.

LIU S., LIU H., LIN L., YUAN Y., DAI F. Y Z. ZHUANG. (2012). Development of 20 Microsatellite Markers for Solenocera crassicornis and Their Cross-Species Application in Solenocera melantho. International Journal of Molecular Sciences.13: 9218-9224. https://doi.org/10.3390/ijms13079218

MACHADO-SCHIAFFINO G., JUANES F. Y E. GARCÍA-VAZQUEZ. (2011). Identifying unique populations in long-dispersal marine species: gulfs as priority conservation areas. Biol. Conserv. 144 (1): 330-339. https://doi.org/10.1016/j.biocon.2010.09.010

MALAUSA T., GILLES A., MEGLÉCZ E., BLANQUART H., DUTHOY S., COSTEDOAT C., DUBUT V., PECH N., CASTAGNONE-SERENO P., DÉLYE C., FEAU N., FREY P., GAUTHIER P., GUILLEMAUD T., HAZARD L., LE CORRE V., LUNGESCARMANT B., MALÉ P. J., FERREIRA S. Y J. F. MARTIN. (2011). Highthroughput microsatellite isolation through 454 GS-FLX Titanium pyrosequencing of enriched DNA libraries. Molecular Ecology Resources 11: 638-644. https://doi.org/10.1111/j.1755-0998.2011.02992.x

MARCUCCI V. C, GESTO E. S. M., ROGEL B., SAZATORNIL M., BRACCALENTI J. C., SAN_MARTIN_PRATA P. Y P. DE_CARLI. (2017). Diversidad y estructura genética poblacional del langostino argentino (Pleoticus muelleri) mediante el análisis de secuencias de ADN mitocondrial de región control. Revista de Informes Científico Técnicos de la Universidad Nacional de la Patagonia Austral. http://dx.doi.org/10.22305/ict-unpa.v9i3.256

MCMILLEN-JACKSON A. L., BERT T. M. Y P. STEELE. (1994). Population genetics of the blue crab Callinectes sapidus: modest population structuring in a background of high gene flow. Mar. Biol. 118: 53-65. https://doi.org/10.1007/BF00699219

MINAGRI. (2017). Desembarques de pesca marítima. Subsecretaría de Pesca y Acuicultura. Ministerio de Agricultura, Ganadería y Pesca. Nación Argentina, en: http://www.agroindustria.gob.ar/sitio/areas/pesca_maritima/informes/economia/index. php.

MONTES I., CONKLIN D., ALBAINA A., CREER S., CARVALHO G. R., SANTOS M. Y A. ESTONBA. (2013). SNP Discovery in European Anchovy (Engraulisen crasicolus, L) by High-Throughput Transcriptome and Genome Sequencing. Plos One 8: e70051. https://doi.org/10.1371/journal.pone.0070051 
PALUMBI S. R. (1994). Genetic divergence, reproductive isolation, and marine speciation. $\begin{array}{llll}\text { Ann } & \text { Rev } & \text { Ecol } & \text { Syst. }\end{array}$ https://doi.org/10.1146/annurev.es.25.110194.002555

PAZ GARCÍA D. A., MUNGUÍA VEGA A., PLOMOZO LUGO T. Y A. HUDSON WEAVER. (2017). Characterization of 32 microsatellite loci for the Pacific red snapper, Lutjanus peru, through next generation sequencing. Mol Biol Rep. DOI 10.1007/s11033-017-4105-4. https://doi.org/10.1007/s11033-017-4105-4

PERINA A., GONZÁLEZ-TIZÓN A. M., VIZCAÍNO A., GONZÁLEZ-ORTEGÓN E. Y A. MARTÍNEZ-LAGE. (2016). Isolation and characterization of 20 polymorphic microsatellite loci in Palaemon serratus and cross-amplification in Palaemon species by 454 pyrosequencing. Conserv Genet Resour.

PLOUGH L. V. Y P. B. MARKO. (2014). Characterization of Microsatellite Loci and Repeat Density in the Gooseneck Barnacle, Pollicipes elegans, Using Next Generation Sequencing. Journal of Heredity. 105: 136-142. https://doi.org/10.1093/jhered/est064

RATHKE M. H. (1837). Bertragzur Fauna der Krym. Mémoires de l'Académie Impériale des Sciences de St. Petersbourg. 3: 371-380.

SLATKIN, M. (1985). Rare alleles as indicators of gene flow. Evolution 39: 53-65. https://doi.org/10.1111/j.1558-5646.1985.tb04079.x

STEPIEN C. A. Y T. D. KOCHER. (1997). Molecules and morphology in studies of fish evolution. En: Kocher, T.D., Stepien, C.A. (eds.). Molecular Systematics of fishes. Academic Press, San Diego. 1-11.

STOUTAMORE J. L., LOVE C. N., LANCE S. L., JONES K. L. Y D. TALLMON. (2012). Development of polymorphic microsatellite markers for blue king crab (Paralithodes platypus). Conservation Genetics Resources, DOI 10.1007/s12686-012-9668-8. https://doi.org/10.1007/s12686-012-9668-8

THOMPSON J. D., HIGGINS D. G. Y T. J. GIBSON. (1994). CLUSTAL W: improving the sensivity of progressive multiple sequence alignment through sequence weighting, position specific gap penalties and weight matrix choice. Nucleic Acids Res. 22: 46734680. https://doi.org/10.1093/nar/22.22.4673

THORPE K.L., HUTCHINSON T.H., HETHERIDGE M.J., SCHOLZE M., SUMPTER J.P. Y C.R. TYLER. (2000). Development of an in vivo screening assay for estrogenic chemicals using juvenile rainbow trout (Oncorhynchus mykiss). Environ. Toxicol. Chem. 19(11): 2812-2820 pp. En: AQUIRE (Aquatic toxicity Information Retrieval) database. U.S. Environmental Protection Agency, National Health and Environmental Effects Research Laboratory, Mid-Continent Ecology Division, Duluth, Minnesota.

WARD R. D., WOODWARK M. Y D. O. F. SKIBINSKI. (1994). A comparison of genetic diversity levels in marine, freshwater, and anadromous fishes. J Fish Biol. 44: 213232. https://doi.org/10.1111/j.1095-8649.1994.tb01200.x

XIONG L. W., WANG Q. Y G. F. QIU. (2012). Large-Scale Isolation of Microsatellites from Chinese Mitten Crab Eriocheir sinensis via a Solexa Genomic Survey. International Journal of Molecular Sciences. 13: 16333-16345. https://doi.org/10.3390/ijms131216333

ZANE L., BARGELLONI L. Y T. PATARNELLO. (2002). Strategies for microsatellite isolation: a review. Mol. Ecol. 11: 1-16. https://doi.org/10.1046/j.0962$\underline{1083.2001 .01418 . \mathrm{x}}$ 